\title{
Evaluation on Ecological Environment of City Based on Entropy and Dissipative Structure Theory_-In Case of Dangyang in Hubei Province*
}

\author{
Ximing Sun, Xia Liu \\ Wuhan Institute of Technology, Wuhan, China \\ Email: 1x421686243@sina.com
}

Received October 12, 2012; revised November 15, 2012; accepted December 17, 2012

\begin{abstract}
Evaluation on ecological environment system is to describe and assess the quality of ecological environment, and accurately reflect the ecological environmental quality and pollution situation. In this paper, after reviewing the domestic and foreign relative researches, from the theory perspective of the dissipative structure of complex system, we analyzed the city ecological system dissipative structure characteristic, and discussed the process forming dissipative structure, then we constructed the city ecosystem entropy parameter table and entropy evaluated model. The total entropy dynamic, scientifically reflects the city ecology system level and developing trend, so in fact it provides the theory basis and method to improve the overall quality of ecological environment, finally we took Dangyang as a case study, and got the same conclusion with the actual situation.
\end{abstract}

Keywords: Entropy; Dissipative Structure; Evaluation; Index System

\section{Introduction}

City ecological system is the important carrier of human society and economic development, and is the closest system related to the human life. Nowadays, due to rapid population growth, rapid economic development as well as the irrational exploitation and utilization, ecological balance is being destroyed, and the ecological environment is deteriorated, which seriously troubled the city development. So how to effectively control the deterioration of the city' ecological environment, and how to attain sustainable development have became a popular issue of city' development. And to carry out the evaluation of city' ecological environment is to grasp the suitability and its variation trend, and reveal the reason of the ecological environment' damage or degradation, then seek the method and way to improve and enhance the quality of ecological environment, which provides a scientific basis and policy support for ecological environment protection, and finally stimulate coordinated development on region economy, resources and environment.

\section{The Current Situation of Domestic and Foreign}

\subsection{The Foreign Research Status}

The research on international ecological environment

\footnotetext{
${ }^{*}$ Fund: Soft Science Research Project of Hubei Province (2011DEA013).
}

quality assessment started earlier, but until recently, the part becomes the research hot spot. At present, foreign country has divided the evaluation of ecological environment into different scales, such as the United States, they have established a number of ecological environmental monitoring and assessment program. In early 90the United States Environmental Protection Agency carried out the study on ecological environment monitoring and evaluation of the project (EMAP), the project initially was from the national scale to evaluate the ecological quality, and did long-term monitoring, then they carried out environmental monitoring and evaluation (REMAP) to the state unit. In the quality assessment of ecological environment, the foreign tended to regard the sustainable development of a region as the standard, and put forward the evaluation index system. For examples, the Hebrew University Jerusalem proposed human activity intensity index, the United Nations department founded the human development index, Daly and Cobb developed the sustainable development model of economic welfare, Canadian International Development research institute put forward the sustainable development of environment and economy model, and the sustainable development committee put forward the index system of sustainable development, the United States EMAP put forward assessment index of life cycle and so on. 


\subsection{The Domestic Research Status}

China's ecological environment quality evaluation began to cause the attention of people in the late nineteen eighties to the early 90 's, the comprehensive index system research also emerged as the times required, it focused of agricultural ecosystem evaluation, followed by the city quality assessment of ecological environment, then related to regional environmental planning, and the ecological environment of the mountain area, and comprehensive evaluation of Provincial Ecological environmental. In the research of indexes system, from a biological point of view, Liao Jiwu, Sun Wu and Yin Qiuju, selected biomass and growth index to evaluate the ecological quality of Hainan Island, Zheng Xinqi, Wang Aiping, used the theory of landscape ecology and the RS, GIS technology to establish regional ecological environment quality evaluation index system. Peng Buzhuo emphasized that the ecological environment evaluation should be along with the change of time and space to do corresponding change on the evaluation method and index system of regional ecological system, according to actual situation of Baiyin, Jia Yanhong used AHP method to determine the ecological environmental quality evaluation index system, and based on the evaluation factors weight and classification quantization, they used comprehensive evaluation model to evaluate ecological environment quality in Baiyin area [1-3].

As everyone knows, city ecological system is an extremely complex, multivariate and multi-level system, the evaluation of ecological environment is the interaction process of subjective and objective analysis. Traditional research methods were mainly static and linear technology, and it is difficult to bear on the research of ecological environment. In this paper, it was based on the theory of systematic science research (complexity theory and related outcomes of uncertainty theory), combined qualitative and quantitative analysis to study city' ecological system and its subsystems, it could provide fundamental information for the management and decision-making of city ecological system.

\section{Dissipative Structure of City Ecosystem}

\subsection{The Theory of Dissipative Structure}

Prigogine (Prigo gign) presented dissipative structure theory based on open system, he used the non balanced perspective, to study the entropy phenomenon in organic and inorganic, social and economic system, and pointed out that, in open systems, due to the negative entropy flows, it can compensate the increase of positive entropy of internal system (Neh Corris, Prigogine, 1992).

Entropy theory is the research about the evaluation of system effectiveness, it dynamic reflect system evolution process and development trend, in which, the increase of entropy reveal that the closed system irreversible caused the disorder and low efficiency due to interaction affection of each element, in the same time, dissipation theory reveals that a open system which is far from equilibrium state, through material and energy exchange process with external environment, open system will be from initial chaos state to the stable and orderly organizational structure and system efficiency is increasing.

And according to the second law of thermodynamics and entropy equation of Clausius, $d S>0$, the open system entropy equation is established:

$$
S_{A}=S_{I}+S_{E}
$$

where $S_{A}$ is the total entropy of whole system, it is the comprehensive decision by the internal system entropy $S_{I}$, and entropy flow $S_{E}$ from the external environment. The $S_{E}$ is generally interpreted as negative entropy, which can be positive or negative. $S_{I}$ refers that, for a closed system, because the system self complexity and the complexity of the environment, the evolution is full of uncertainty and chaos, which leads to the system in diminishing energy, and develop irreversibly from order to disorder, this is the increase process of entropy, $S_{I}$ is positive permanently. Only when $S_{A}<0$, the dissipative structure formates. So the total entropy reveals the disorder degree, entropy is larger and positive, the more disordered. Entropy is negative and small, the better state. At the same time, entropy changes also reveals a system development direction and extent, $\Delta S$ is positive, it express entropy increase and indicate that ecosystem decline, $\Delta S$ is negative, the entropy become low, it express that environment system improve and get better development [4-7].

Dissipative structure theory points out, system from disorder transition to the dissipative structure has some necessary conditions. A system must be open, namely the system must have material, energy and information exchange with the surrounding environment; the second is that the system must be far away from the equilibrium state, the equilibrium state and near equilibrium cannot occur mutation to makes the system from disorder to order. Thirdly, the relationship between the different elements of system is non linear.

\subsection{Dissipative Structure Characteristics of City Ecosystem}

First, city ecological system is an open system, mainly in the exchange with natural environment and social environment. The former include it draw air, water and raw materials from nature, and at the same time, discharge the waste. Also, it is mainly due to the modern social division of labor, division of labor contributed to the development of product and the change of social structure, and led to the necessity of exchanges. Second, each en- 
terprise, each department within the city are restricted and promoted mutually, exhibiting nonlinear interaction. In the case of steel and iron industry machinery industry, development of steelmaking make steel industry have more raw materials so as to promote the development steel increase, when it increases, it creates more advanced equipment, in turn, it will promote the development of machinery industry, this is a kind of complex positive feedback effect. Of course, because the environment is crowded and price will decrease with the increase of the products, these factors will restrict on iron and steel industry development. The role of mutual promotion and mutual restriction show a variety of complex structure. In third, due to the nonlinear interaction between the system and the outside, and full of material, energy and information exchange, city system is generally in a non-equilibrium state, shows that city are full of the flow of "force", it must rely on the outside of the material and energy supply to maintain the orderly steady state [1-3].

To sum up, the city is with the above features, in line with the dissipative structure theory, so it can apply dissipative structure theory to analyze the evolution process of the city ecological system.

\section{Evaluation Model on City Ecosystem Dissipative Structure Entropy}

City ecosystem entropy evaluation ideas is that under the guidance of the dissipation entropy theory, through the construction of performance evaluation system, then measure the entropy, according to entropy changes, it comprehensively, dynamic reflect evaluation object state and development trend. Its purpose is to discover the city ecosystem factors, and take measures to minimize the increase of entropy of open system, and enable the system to the negative entropy, and order development.

\subsection{City Ecosystem Evaluation Index Selection}

From the actual need to reflect the city ecological environment, in the basis of using existing research results, apply method of theory to design evaluation index system of ecological environmental, then through expert screening, computer simulation, to establish the weight of every index, and through entropy correction to constitute a complete evaluation index system.

\subsection{Construction City Ecological System Entropy Evaluation Model}

$$
\begin{gathered}
D_{s}=\sum_{i=1}^{m} w_{i} d_{s i} \\
d_{s i}=-k_{\mathrm{B}} \sum_{j=1}^{n} p_{i j} \ln p_{i j} d_{s i}
\end{gathered}
$$

$$
\begin{gathered}
K_{B}=(\ln m)^{-1} \\
p_{i j}=\frac{X_{i j}}{\sum_{j=1}^{n} x_{i j}}
\end{gathered}
$$

In the formula (1), $i$ are the subsystems namely the main influencing factors of the city ecosystem $(i=1,2 \cdots$, $m) ; w_{i}$ is the weights of various subsystems, given the weight is difficult to calculate objectively, so through the expert group decision method, establish the weight of evaluation set, then using information entropy correction method ultimately determine the weight; $D_{S}$ is the total entropy of the open system.

In the formula (2), $d_{s i}$ is the subsystem entropy value, $j$ are influencing factor of each subsystem performance. When the impact factor is the internal factors, formula (2) is positive; when the influencing factors system of external factors, is the system continuously from the external environment into energy and material, formula (2) is negative or positive.

In the formula (3), $K_{B}$ is subsystem entropy coefficient.

In the formula (4), $p_{i j}$ is probability that main factor of subsystem influence on dst, $x_{i j}$ is the correction value of affecting factor, $\mathrm{n}$ is factor number of subsystem impact [1-3].

\section{Empirical Study on the Evaluation of City Ecological System}

This paper took Dangyang city as an example, according to Table 1 to establish city ecological system evaluation index system, index data sample values are derived from the "Hubei City Statistical Yearbook", in the base of comprehensive expert determination of weights and using the parameter standard data to correct the sample values, Then apply entropy evaluation model, obtain city ecological system entropy, as is shown in Table 2. Considering the timeliness and accessibility of data, parameter standard value refers state city assessment standards, optimum values or good value of domestic city construction. The specific calculation procedure is as follows:

1) Construction the evaluation index system of city ecosystem. According to Table 1, select index sample data, do standardization of sample data, calculate index correction value $X_{i j}$. The correction index value $X_{i j}$ is the outcome that $S_{i j}$ divide the standard value.

2) Determine the weight of subsystem $w_{i}$. Subsystem weight is obtained by Delphi method, because it is subjective, so use information entropy correction method can be evaluated with dynamic changes of object and automatically generate the desired objective weight, eliminating the subjective factors the effect of. 
Table 1. Parameter table of city ecosystem total entropy.

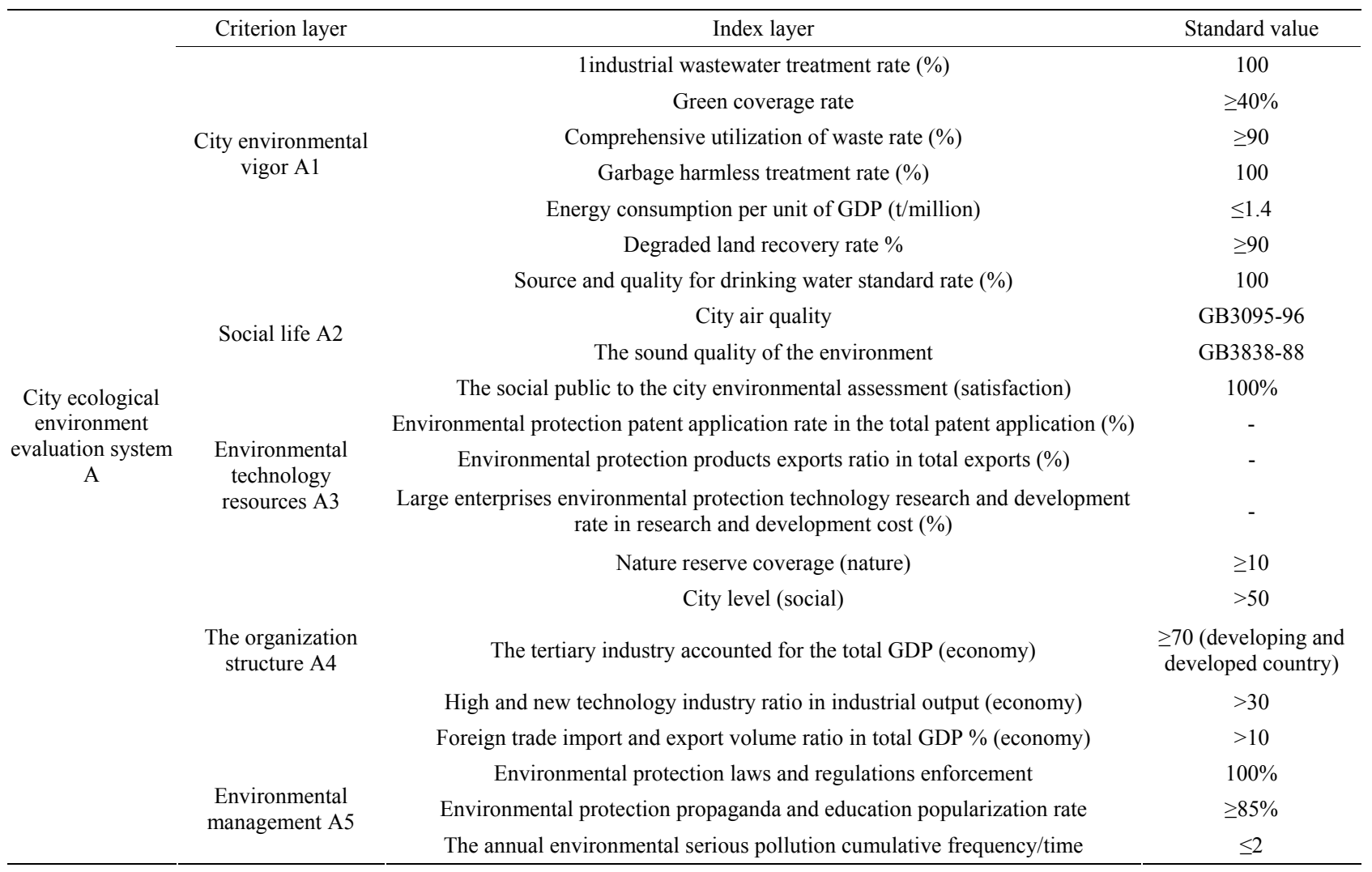

If there are $M$ indicator, expert give each indicator $n$ evaluation value, says $a_{i j}$ is the $J$ value of the $I$ index, index value matrix is $A=\left(a_{i j}\right) m \times n$, entropy weight formula is as follows:

$$
\begin{gathered}
p_{i j}=\frac{a_{i j}}{\sum_{j=1}^{n} a_{i j}}, \sum p=1, i=1,2, \cdots, m ; j=1,2, \cdots, n \\
\mathrm{e}_{i}=-k \sum_{j=1}^{n} p_{i j} \ln p_{i j}, k=\frac{1}{\ln n}, 0 \leq \mathrm{e}_{i} \leq 1
\end{gathered}
$$

So:

$$
w_{i}=\frac{1-\mathrm{e}_{i}}{m-\sum_{i=1}^{m} \mathrm{e}_{i}}
$$

As can be seen, if an index entropy is more small, show its index variation is more big, the amount of information.

3) Computing contribution rate of subsystem. Contribution rate of System for total entropy change $=$ subsystem entropy change in volume/total system entropy variation.

From Table 2: the change of total entropy value:

$$
D=D 2010-D 2007=(-0.886)-(-0.772)=-0.114<0
$$

The relative change amount of entropy

$$
=(-0.114) /(-0.772) \times 100 \%=14.76 \%
$$

Contribution rate of city environmental vigor subsystem:

$$
[(-0.98)-(-0.72)] /(-0.114)=2.28
$$

Contribution rate of environmental management:

$$
[(-0.94)-(-0.898)] /(-0.114)=0.368
$$

So total entropy value decrease 0.114 , its change rate reached $14.76 \%$, it showed that the ecological system of Dangyang city has been greatly improved, in which the environmental vigor system for the enhancing of systematic evaluation is maximum, followed by raising of management level, and as a result of environmental little protection patent number, low production efficiency, the level of science and technology need to be improved, and block the whole city ecosystem evaluation.

The Dangyang city ecosystem system increase, mainly express in the:

1) City had invested more 10 billion to improve ecological environment, develop ecological agriculture and project "becoming rich by green". They had built 100 million efficient forestry base (30 million mu of fastgrowing forest, 20 million acres of efficient economy forest, 50 million acres of short-rotation industrial raw material forest), 10 million acres of pollution-free vegetable base, 10 million acres of fish breeding base. After 
Table 2. Measurable table of city ecosystem evaluation entropy.

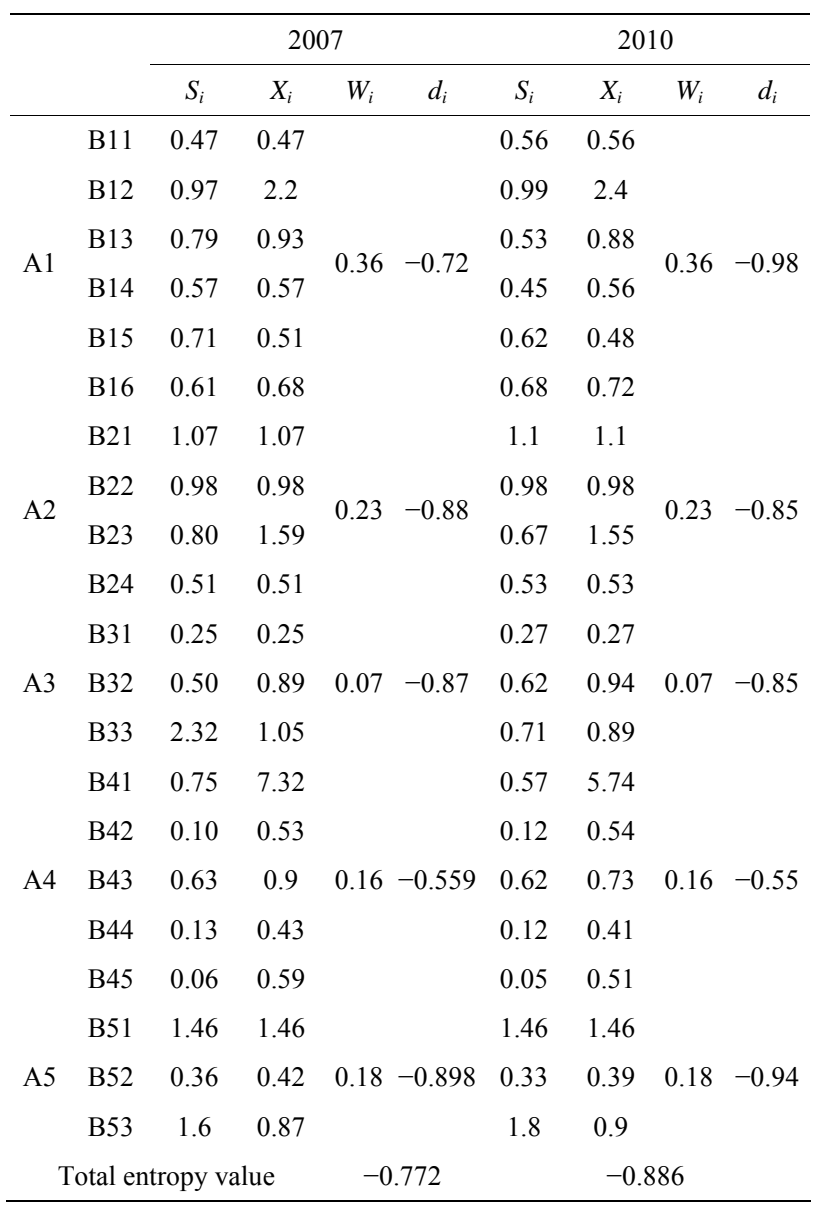

efforts, they also will strive for 230 million special loan for the ecological environment construction, afforestation 2.19 million mus. Through construction, it effectively eased the situation of soil erosion, and improved the ecological environment.

2) Dangyang city compiled (city ecological construction planning) as the basis for strengthening the ecological construction, and actively promoted the construction of environmental protection, and strengthened their perceptual knowledge and increasing the spontaneous ecological construction enthusiasm of public, which promoted the city's ecological construction.

\section{Summary}

The study evaluation method was used for the two annual ecological environment quality evaluation of Dangyang city, the results are almost the same as the true feelings of researcher and local people on the evaluation of ecological environment quality, the ecological environment entropy value objectively reflects the true situation, also reflects the city ecological environment spatial variation characteristics and the time transformation trend. It conduces to analysis of variable factors, and has important reference value on the adjustment of development policy and relationship, and strengthening management of ecological environment. Nevertheless in the future, in-depth study of indicator selection and weight assignment of rationality and scientific sex are needed, also it is necessary to improve the evaluation system and test in practice.

\section{REFERENCES}

[1] T. Wei, X. D. Zhu and Y. F. Li, "Ecosystem Health Assessment of Xiamen City Based on Catastrophe Progression Method [J]," Journal of Ecology, No. 12, 2008, pp. 17-22.

[2] T. L. Hu, Z. F. Yang, M. C. He and Y. W. Zhao, "A Assessment Method and Its Application on City Ecosystem Health [J]," Journal of Environmental Science, No. 2, 2005, pp. 10-12.

[3] Y. N. Zhang, "Ecosystem Health Evaluation of the Ji'nan City [D]," Shandong Normal University, Ji'nan, 2011.

[4] P. Y. Ren, L. Zhang and Y. Song, "The Role in Enterprise Organization and Decision Making Based on Complexity Science Management Entropy, Dissipative Structure Theory [J]," Management World, No. 6, 2001, pp. 10-14.

[5] X. X. Wang and P.-Y. Ren, "A New Performance Evaluation Method: Management Entropy Evaluation Model [J]," Modern Management Science, No. 6, 2009, pp. 8185.

[6] X. B. Xiong, "Performance Evaluation of Enterprise Knowledge Management Performance Based on the Management Entropy, Dissipative Structure of Management [J]," Finance and Trade Research, No. 1, 2009, pp. 69-72.

[7] Y. Q. Zhou, "Corporate Diversification Strategy Evaluation Model Based on the Entropy Theory [D]," Zhejiang Business University, Zhejiang, 2007. 